\title{
Using Digital Game, Augmented Reality, and Head Mounted Displays for Immediate-Action Commander Training
}

\author{
https://doi.org/10.3991/ijet.v12i02.6303 \\ H. Mitsuhara \\ Tokushima University, Tokushima, Japan \\ mituharadis.tokushima-u.ac.jp \\ K. Iguchi \\ Tokushima University, Tokushima, Japan \\ c501637009@tokushima-u.ac.jp \\ M. Shishibori \\ Tokushima University, Tokushima, Japan \\ bori@is.tokushima-u.ac.jp
}

\begin{abstract}
Disaster education focusing on how we should take immediate actions after disasters strike is essential to protect our lives. However, children find it difficult to understand such disaster education. Instead of disaster education to children, adults should properly instruct them to take immediate actions in the event of a disaster. We refer to such adults as Immediate-Action Commanders (IACers) and attach importance to technology-enhanced IACer training programs with high situational and audio-visual realities. To realize such programs, we focused on digital game, augmented reality (AR) and headmounted displays (HMDs). We prototyped three AR systems that superimpose interactive virtual objects onto HMDs' real-time vision or a trainee's actual view based on interactive fictional scenarios. In addition, the systems are designed to realize voice-based interactions between the virtual objects (i.e., virtual children) and the trainee. According to a brief comparative survey, the AR system equipped with a smartphone-based binocular opaque HMD (Google Cardboard) has the most promising practical system for technology-enhanced IACer training programs.
\end{abstract}

Keywords-Augmented reality, digital game, disaster education, head mounted display, interactive learning

\section{Introduction}

We are always in danger of natural and human-caused disasters. For example, earthquakes and terrorist attacks are extremely dangerous because they occur with no prior warning. Forecasting these disasters is difficult with even the most advanced 
technologies. Therefore, disaster education focusing on the execution of planned immediate actions against disasters, which has been conducted in various ways and scenes, is essential to protect our lives. For example, evacuation drills are frequently conducted in schools, companies, and communities. Thus, it is expected that we have acquired sufficient knowledge to survive disasters. However, such expectation differs from reality. The occurrence of a disaster is frequently regarded as someone else's problem, which means that disaster education has not been established yet. For example, in many evacuation drills, participants simply follow fixed evacuation routes under the usual conditions and cannot feel a sense of tension. In other words, conventional evacuation drills are not realistic. Nowadays, disasters are diversified and we must acquire sufficient knowledge from these practical examples. Although we have acquired knowledge, we occasionally misesteem a real disaster in front of our eyes and do not consider evacuating the place immediately (i.e., normalcy bias). We do not always apply knowledge to immediate actions against a real disaster. Because of these multiple reasons, disaster education is difficult to be established.

Preferentially, disaster education should be established for children because they are most vulnerable to disasters. However, compared to adults, children find it difficult in applying knowledge to a real disaster. Rather than being subjected to normalcy bias, children may panic and feel incapacitated by fear. Therefore, adults should appropriately instruct children to take immediate actions in the event of a disaster. We refer to such adults as Immediate-Action Commanders (IACers). In particular, school teachers should be trained to become IACers because they have a responsibility to protect the lives of numerous students. However, few satisfactory training programs are currently available, and majority of adults have not been trained yet. In this context, we must develop multiple IACer training programs.

IACer training programs must represent disasters realistically because trainees (e.g., school teachers) find it difficult to imagine disaster situations as they do not have a prior experience of such an event. In other words, the programs need situational and audio-visual realities. An approach to the realistic representation is to integrate digital technologies into the programs, i.e., technology-enhanced IACer training programs. In a variety of digital technologies, digital games with high interactivity and audio-visual effects have gathered significant attention in disaster education [1]. For example, there have been simulation games that allow players to evacuate from a virtual 3D building [2][3]. Djordjevich et al. [4] developed an emotional agentinvolved simulation game that trains disaster responders (incident commanders) to look at disasters on a macro scale. Wahyudin and Hasegawa [5] developed a 3D roleplaying game that trains disaster volunteers to make appropriate ethical decisions. These games aim at improving situational and audio-visual realities by scenarios and virtual worlds (computer sounds and graphics) respectively.

From our perspective, technology-enhanced IACer training programs should involve gaming technologies and the real world to further improve the situational and audiovisual realities. In particular, we focus on visual reality because virtual worlds may not encourage trainees to feel a sense of tension due to representational limitation in computer graphics $(\mathrm{CG})$. For high visual reality, we considered implementing augmented reality (AR) that superimposes interactive virtual objects (e.g., 3DCG characters) on a real-time vision. 


\section{Training of Immediate-Action Commanders}

Disaster responders take life-saving actions. Specialists (e.g., rescue teams) sometimes rush into the site and citizens sometimes try to save lives on the scene. In either case, disaster responders start taking actions after a disaster strikes. To save lives of numerous children, majority of the adults should be trained to become IACers.

\subsection{Ideal Training Program}

Appropriate immediate actions will differ depending on the type of disasters. During an earthquake, for example, children should move away from windows and keep huddling under tables until the tremors stop. For children to take appropriate immediate actions, IACers must instruct children to take the proper actions.

From our perspective, an ideal IACer training program should satisfy the following requirements. Fig. 1-a schematically shows the ideal program.

(1) Conducted in familiar (usual) spaces for trainees: This requirement can improve situational reality wherein training situations are closer to probable disaster situations. For example, a familiar space for school teachers is their classroom. In other words, the program can be conducted anywhere.

(2) Involving real children: In a real disaster, children will exhibit various reactions. Based on these reactions, adults will instruct the children to take necessary actions. In particular, vocal commands can be transferred most quickly in disasters. Therefore, the program should involve real children who can exhibit pseudo reactions (e.g., appropriate, inappropriate, or no immediate actions) and follow the vocal instructions of the trainees. This requirement can improve the situational and audiovisual realities.

It is easy to satisfy (1) because special equipment is not necessary. On the other hand, it is difficult to satisfy (2) because it is difficult for children to imagine disaster situations and exhibit pseudo reactions. Therefore, the ideal IACer training program must be redesigned to satisfy (2).

\subsection{Redesigning Ideal Training Program as AR System}

We redesign the ideal program as a technology-enhanced IACer training program. In other words, we aim at satisfying (2) from the viewpoint of digital technology. In the redesign, there are two key issues: "What can be substituted for the real children?" and "How do we introduce a game element?" As the substitute, we adopt AR-based interactive virtual children (i.e., 3DCG characters) who exhibit reactions and take immediate actions against disasters. This means that an AR system is necessary to superimpose the virtual children on a real-time vision (i.e., a trainee's view). As the game element, we adopt a simple interactive fiction (IF) based on voice recognition. We define the following requirements for the AR system. Fig. 1-b schematically shows the redesigned ideal program. 
(1) Working on portable computers: Although recent AR systems can work on various computers (e.g., desktops, laptops, tablets, and smartphones), the ideal AR system should work on computers that are not fixed stations, i.e., using desktop computers should be avoided.

(2) Having interactivity and visual consistencies optimized for head-mounted display: The virtual children should change their reactions according to a trainee's vocal commands. In other words, the AR system should realize voice-based interactions between the virtual children and the trainee using voice recognition techniques. The interactions (i.e., changes in reactions), which are controlled according to branched IF scenarios, can be regarded as the game element. General AR systems aim at improving visual reality in terms of visual consistencies (e.g., geometric and photometric consistencies) while being optimized for visual output devices (i.e., displays). From our viewpoint, head-mounted displays (HMDs) are most suitable to maximize visual reality because HMDs can provide high immersion. Usually, vocal commands will be accompanied by gestures for facilitating the transmission of the commands. HMDs may not excessively encumber trainees' gestures. Therefore, we adopt HMDs for the AR system.

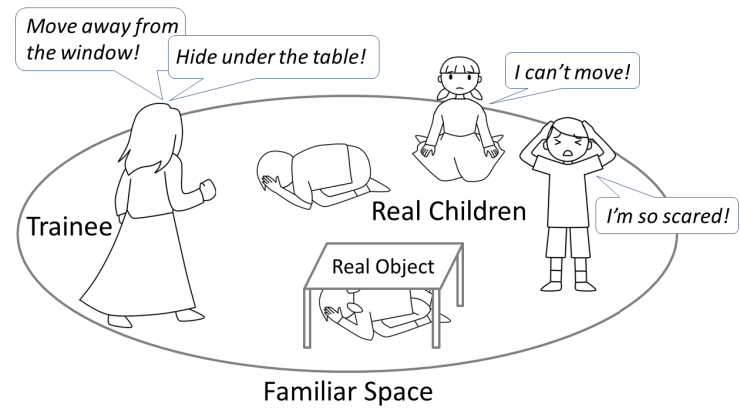

(a) Ideal program

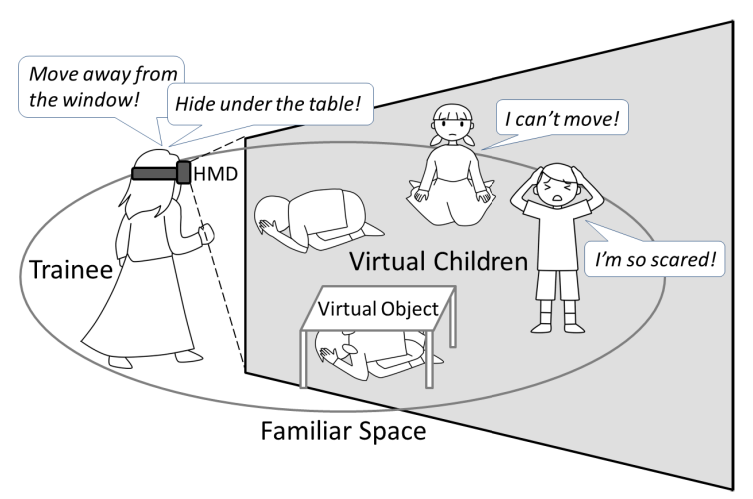

(b) Redesigned ideal program

Fig. 1. Ideal Immediate-Action Commander (IACer) training programs 


\subsection{Related Work}

There have been many educational AR systems [6][7]. Additionally, HMDs have been actively adopted in educational AR systems, for example, training in crossorganizational communication [8] and information exchange in forensic investigation [9]. This is because, nowadays, HMDs are rapidly being advanced, diversified, and commonly marketed. For example, Oculus Rift is a dominant product of binocular opaque HMDs, whereas Google Glass is a long-awaited product of optical seethrough HMDs (i.e., smart glasses). Reiners et al. [10] use Oculus Rift so that workers can learn health and safety risks more realistically in the virtual environment. Suarez et al. [11] use Google Glass so that learners can make instant inquiries with handsfree interaction (voice interaction). In disaster education, Wang et al. [12] developed a $3 \mathrm{D}$-simulator that allows HMD wearers to evacuate from a virtual building fire. We developed evacuation drill systems using AR and HMDs [13][14][15]. These systems superimpose virtual disaster situations represented by 2D/3DCGs or digital hazard maps onto the HMDs' real-time vision so that the participants feel a sense of tension in evacuation.

In simple terms, AR is the integration of virtual worlds with the real world. AR systems for disaster education frequently realize this integration as a mixed reality game $(\mathrm{MRG})$ or an alternate reality game (ARG) that works on portable computers (e.g., smartphones and tablets). For example, Fischer et al. [16] developed a geofencing MRG, where field players can learn disaster response coordination by observing the virtual disaster situations (e.g., radioactive pollution areas) on a digital map. Meesters and Van de Walle [17] developed an ARG, where field players can learn disaster information management (e.g., rescue operations) while carrying out missions presented on portable computers and interacting with volunteer actors who play roles (e.g., victims and police officers) in virtual disaster situations.

\section{Prototype Systems}

Multiple programs (systems) are necessary to establish IACer training. Therefore, using three different types of HMDs, we prototyped a different AR system for each.

\subsection{Overall Design}

We adopted a binocular opaque HMD (Oculus Rift), a smartphone-based binocular opaque HMD (Google Cardboard), and an optical see-through HMD (Epson Moverio). We refer to the AR systems developed for Oculus Rift, Google Cardboard, and Moverio as System-1, System-2, and System-3, respectively.

Oculus Rift provides high immersion $\left(110^{\circ}\right.$ viewing angle and sensitive head motion tracking using acceleration, gyro, and geomagnetic sensors); however, it requires a high-performance computer and an external camera. For System-1, we considered using laptops (e.g., Intel Core i5, 8GB memory, Intel HD Graphics 4600, and Microsoft Windows 8) and a stereoscopic camera (Ovrvision). System-2 works on stand- 
ard smartphones that are placed into a cardboard frame with two lenses. For System2, we considered using Android smartphones (e.g., $2.5 \mathrm{GHz}$ quad-core processor, $2 \mathrm{~GB}+16 \mathrm{~GB}$ memory, and Android 4.4). System-3 works on a dedicated Android device (dual-core processor 1.2 GHz, 1GB + 8GB memory, and Android 4.0) with its connectivity solely restricted to Moverio. All the systems need a microphone to realize voice-based interactions.

System-1 and System-2 superimpose virtual objects (interactive virtual children and visual effects) onto the real-time vision captured by Ovrvision and the smartphone's built-in camera, respectively. System-3 superimposes virtual objects semi-transparently onto a trainee's (i.e., wearer's) actual view. For System-2 and System-3, we considered using a visual marker-based AR library (Vuforia); for these systems, visual markers must be set in the training spaces. For System-1, we implemented a markerless AR using Oculus Rift's sensors and software utilities. Fig. 2 is a schematic of the overall design of the AR systems.

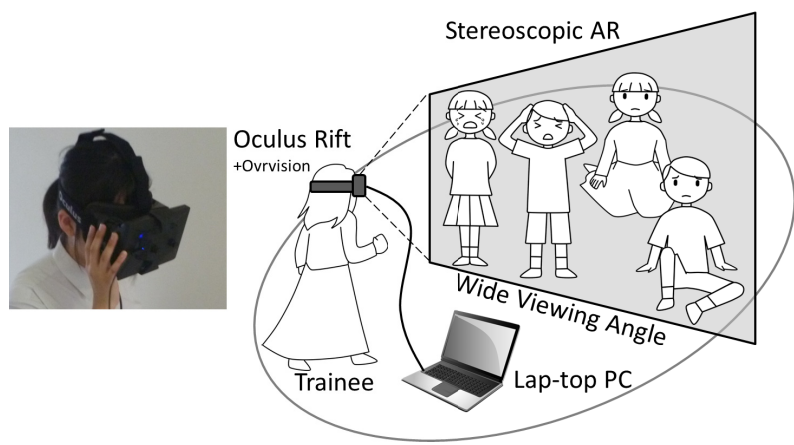

System-1 (Oculus Rift)

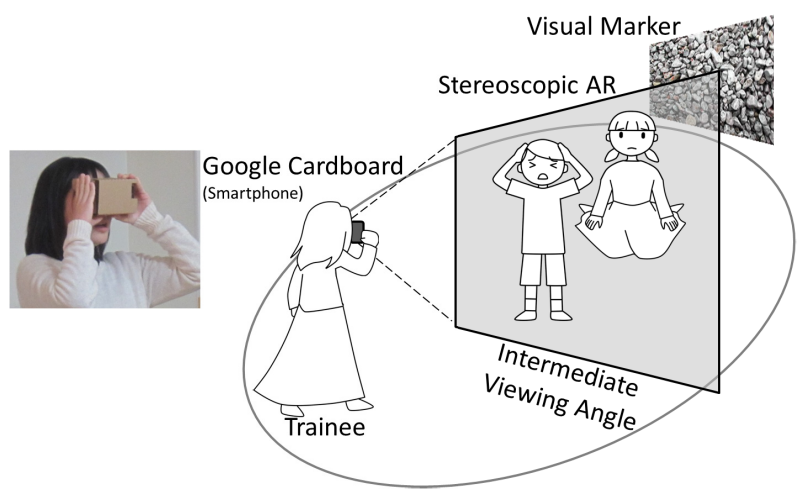

System-2 (Google Cardboard) 


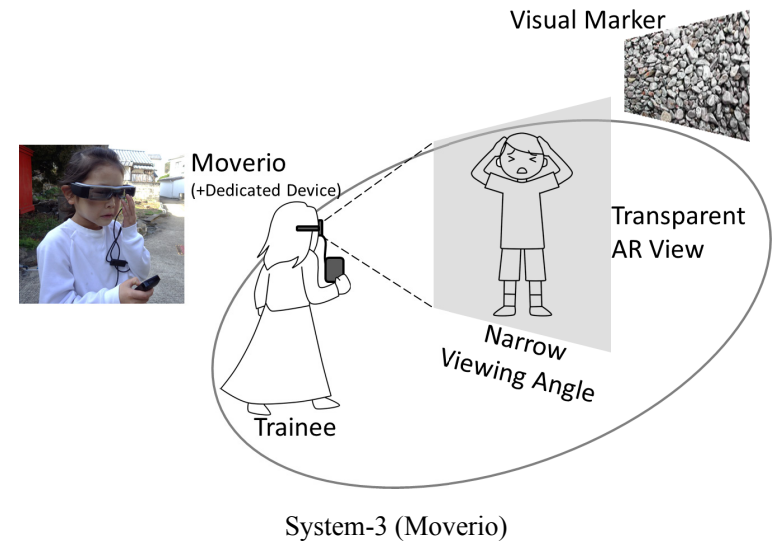

Fig. 2. Three AR systems for IACer training

\subsection{Interactivity}

Interactivity (the game element) in all the systems is realized as voice-based interactions controlled according to a branched IF scenario, which provides multiple endings. The scenario comprises segmentalized disaster situations. Each situation corresponds to a predefined CG animation (with sound effects) of virtual objects and basically has two directional links to the next situations. The next situation depends on whether a trainee says an appropriate command. Fig. 3 shows a structural example of a branched IF scenario.

There are the following interaction modes that correspond to two training difficulty levels. Trainees can choose the mode before the training begins.

(1) Canon Mode (Easy Mode): In this mode, virtual objects (CG animation) and a proper command (e.g., Say, "Move away from the window!") are superimposed for each situation. Trainees only have to say the superimposed command while observing the virtual disaster situation.

(2) Reasoning Mode (Difficult Mode): In this mode, virtual objects for each situation and a fixed message ("Say a proper command.") are superimposed. Trainees have to reason about a proper command and say the command while observing the virtual disaster situation.

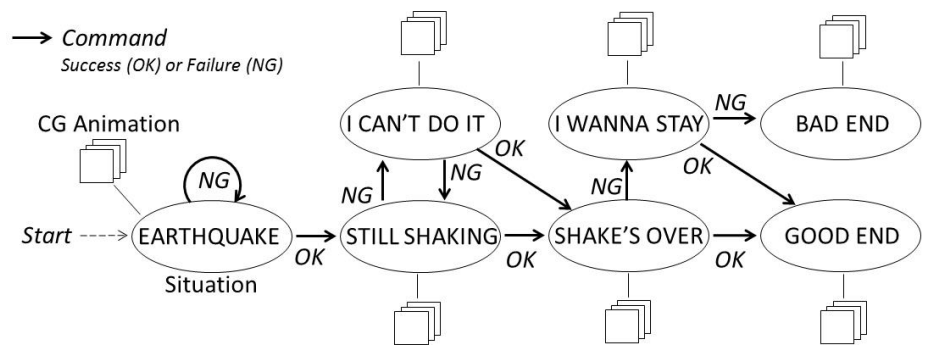

Fig. 3. Branched IF scenario 


\subsection{User Tasks}

For all the systems, users (trainers or trainees) must complete the following tasks to conduct the training program.

(1) Writing IF Scenario: A branched IF scenario is represented as the dataset is divided by a number of situations. Each situation comprises a proper command, next situation (success), and the next situation (failure). To improve the situational reality, users must consider the realistic flow in the branched IF scenario.

Trainees must precisely say the appropriate command corresponding to each situation to reach a good end. An example of the dataset (the scenario shown in Fig. 3) is shown as follows:

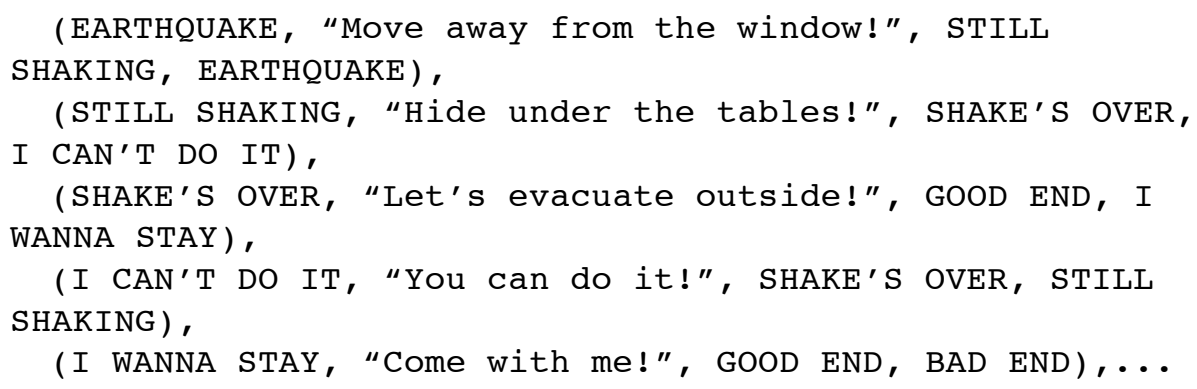

Many users will have difficulty in modeling the virtual objects including CG animations. Currently, we predefine the situations and model the corresponding virtual objects beforehand. Therefore, the users only have to choose the situations and make the realistic flow.

(2) Preparing Visual Markers: The visual markers are used as the spatial scale to superimpose the virtual objects. For System-2 and System-3, users must prepare visual markers to a number of spaces to conduct the training-print out and set them to the spaces. The visual markers should be sufficiently larger so that the systems can be used in wider spaces - clearly capture the visual markers even when trainees stay away from the markers.

(3) Adjusting Virtual Objects: To further improve the visual reality, all the systems should superimpose the virtual objects with high visual consistencies. For high geometric consistency, users must adjust the positions and sizes of the superimposed virtual objects on the basis of the visual markers.

\section{$4 \quad$ AR Views}

We prototyped all the systems using a game engine (Unity) that enables implementers to model 3DCG and program its animations on an integrated development environment. All the systems satisfy the requirement (1); however they have not completely satisfied the requirement (2). This is because they have not realized voicebased interactions (changes in virtual children's reactions according to IF scenarios) and high photometric consistency. In contrast, voice recognition (voice to text) has 
Paper-Using Digital Game, Augmented Reality, and Head Mounted Displays for Immediate-Action Commander Training

been implemented in System-2. Therefore, at this time, the virtual children take fixed actions as time proceeds.

\subsection{System-1 (Oculus Rift)}

System-1 realizes a markerless stereoscopic AR that adjusts the positions of the superimposed virtual objects according to the feedback from the head tracking functions. System-1 superimposes not only virtual children (up to 16 children) but also other virtual objects (e.g., desks) on the real-time vision, taking the advantages of Oculus Rift's high immersion. Fig. 4 shows screenshots of System-1 that represent the situations after an emergency earthquake warning sounds in class. The top picture shows the superimposition of the virtual students seated in a classroom and a fixed message, whereas the bottom picture shows that nearly all students have huddled under the desks (i.e., they took the proper immediate action). However, in the bottom picture, there are some students who are still standing (i.e., they did not take the necessary actions). In such a situation, a trainee must repeat the appropriate command again.

\subsection{System-2 (Google Cardboard)}

System-2 realizes a visual marker-based stereoscopic AR that divides the real-time vision into two visions (for left and right eyes) and adjusts the positions of the superimposed virtual objects, even when the visual markers are lost in the real-time vision, by using the Vuforia's tracking function. Basically, System-2 superimposes not only virtual children (up to 8 children) but also other small virtual objects (e.g., fragments of broken glass) on the real-time vision because regular smartphones' cameras do not have a considerably wide viewing angle. Fig. 5 shows screenshots of System-2. In the top picture (divided for the stereoscopic view), two students have huddled under the table that is not a virtual but a real object. System-2 aims at satisfying (2) by the following functions.

(1) Adjusting the superimposed virtual objects: Prior to on-site training, the users can intuitively adjust the positions and sizes of the superimposed virtual objects by swipe (position) and pinch-in or pinch-out (size) operations on the smartphone's display while looking at the real-time vision. This function can improve the visual reality with high geometric consistency.

(2) Converting a trainee's voice to text: The voice recognition function can convert a trainee's voice (recorded from the smartphone's microphone) to text in real time and save the text data available for reflection on training. In the future, the text data will be used for voice-based interactions.

(3) Expressing occlusion through manual settings: Occlusion can be regarded as the depth interference between virtual and real objects. It is very important for AR systems to appropriately express the occlusion for high visual reality, i.e., real objects in the real-time vision should partially hide the superimposed virtual objects. This function can express the occlusion by enabling users to manually set masking objects (size-adjustable cubes) on the real-time vision beforehand. After the training starts, 
the masked parts of the virtual objects are visually eliminated, i.e., the corresponding cubes become transparent. Fig. 5 (bottom picture) shows that a user is checking whether the occlusion is appropriately expressed (around the virtual student's legs).

\subsection{System-3 (Moverio)}

A trainee wearing Moverio (88g headset) can view the real world through the glasses. System-3 realizes a visual marker-based AR that superimposes virtual objects onto the trainee's actual view-it just presents the virtual objects whose peripheral area is filled with black. System-3 aims at improving the visual reality by a distinctive AR representation (i.e., semi-transparent AR). Fig. 6 shows screenshots of System-3. The visual reality can be maximized toward the real world, but will decrease toward the virtual objects. Moverio cannot present virtual objects widely due to its $23^{\circ}$ viewing angle. Therefore, System-3 can be used only to represent distant disaster situations, i.e., present small distant virtual objects.

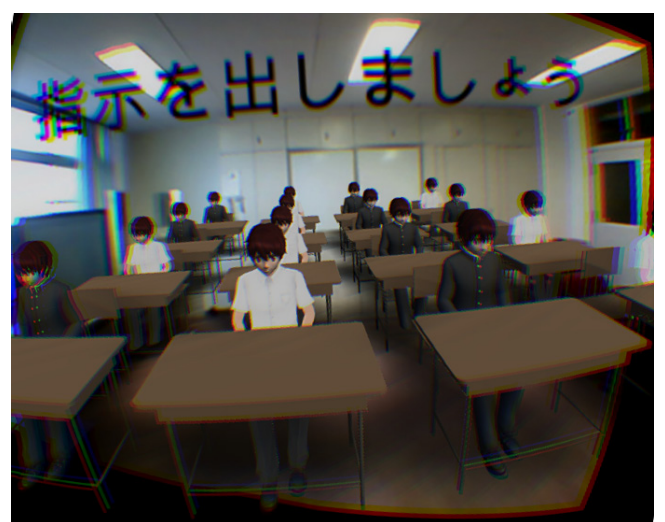

AR view (students seated)

Superimposed message: "Say a proper command."

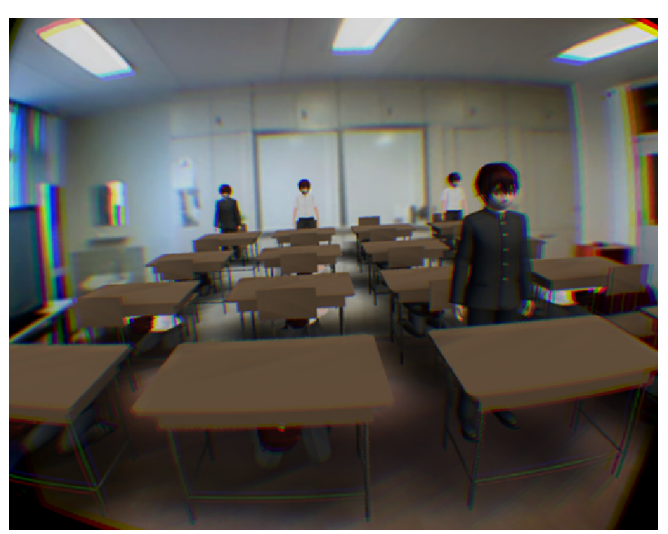

AR view (huddling or standing students)

Fig. 4. Screenshots of System-1 
Paper-Using Digital Game, Augmented Reality, and Head Mounted Displays for Immediate-Action Commander Training

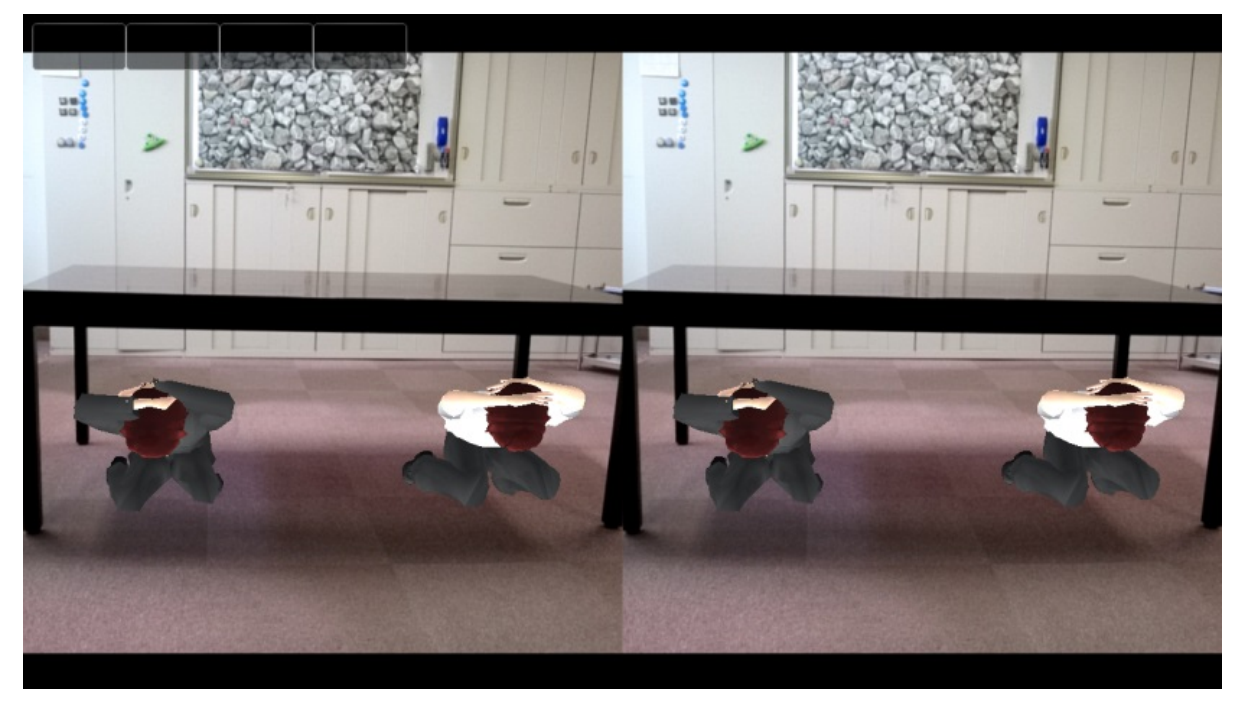

Screenshot (stereoscopis AR view)

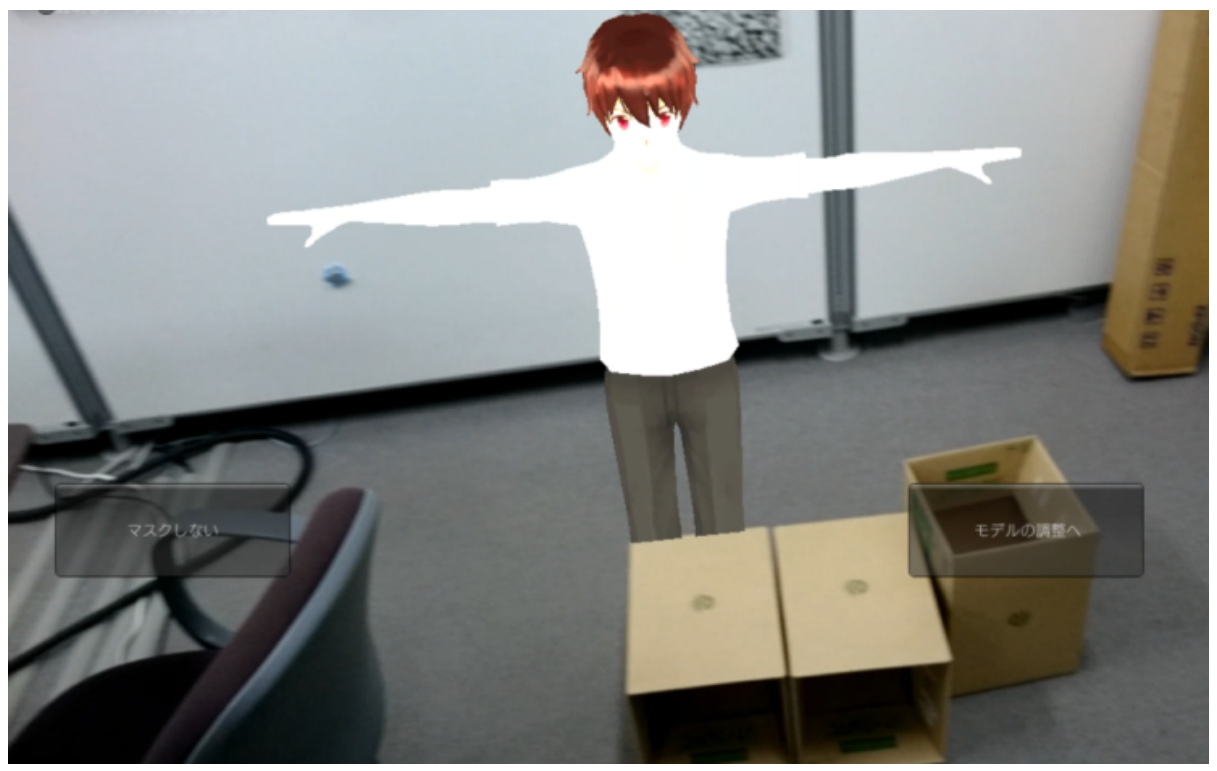

Oculusion expression

Fig. 5. Screenshots of System-2 
Paper-Using Digital Game, Augmented Reality, and Head Mounted Displays for Immediate-Action Commander Training
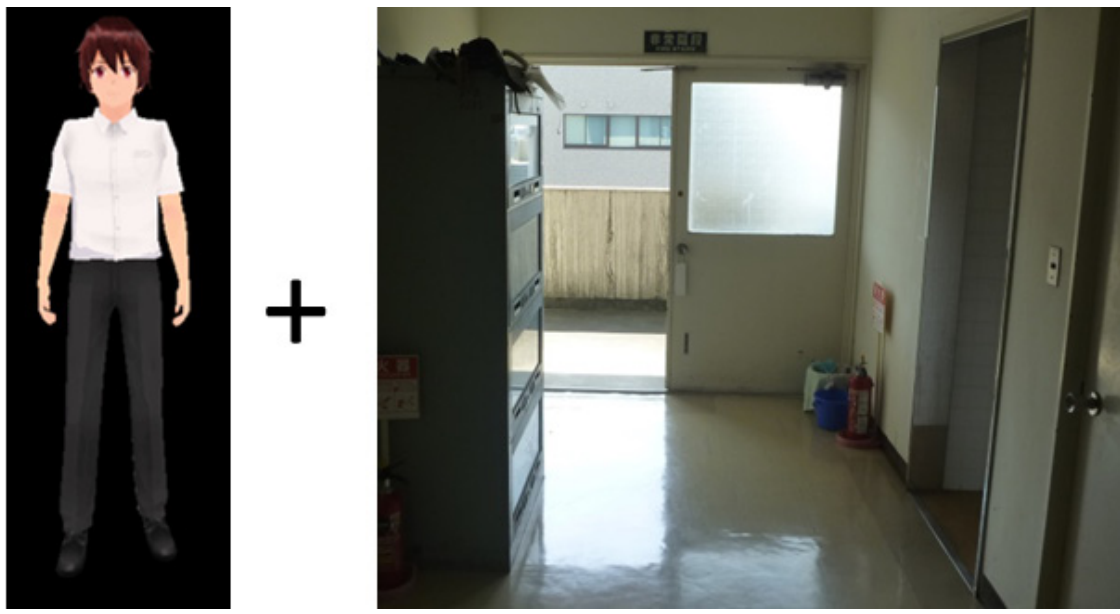

Virtual object (student) User's actial view

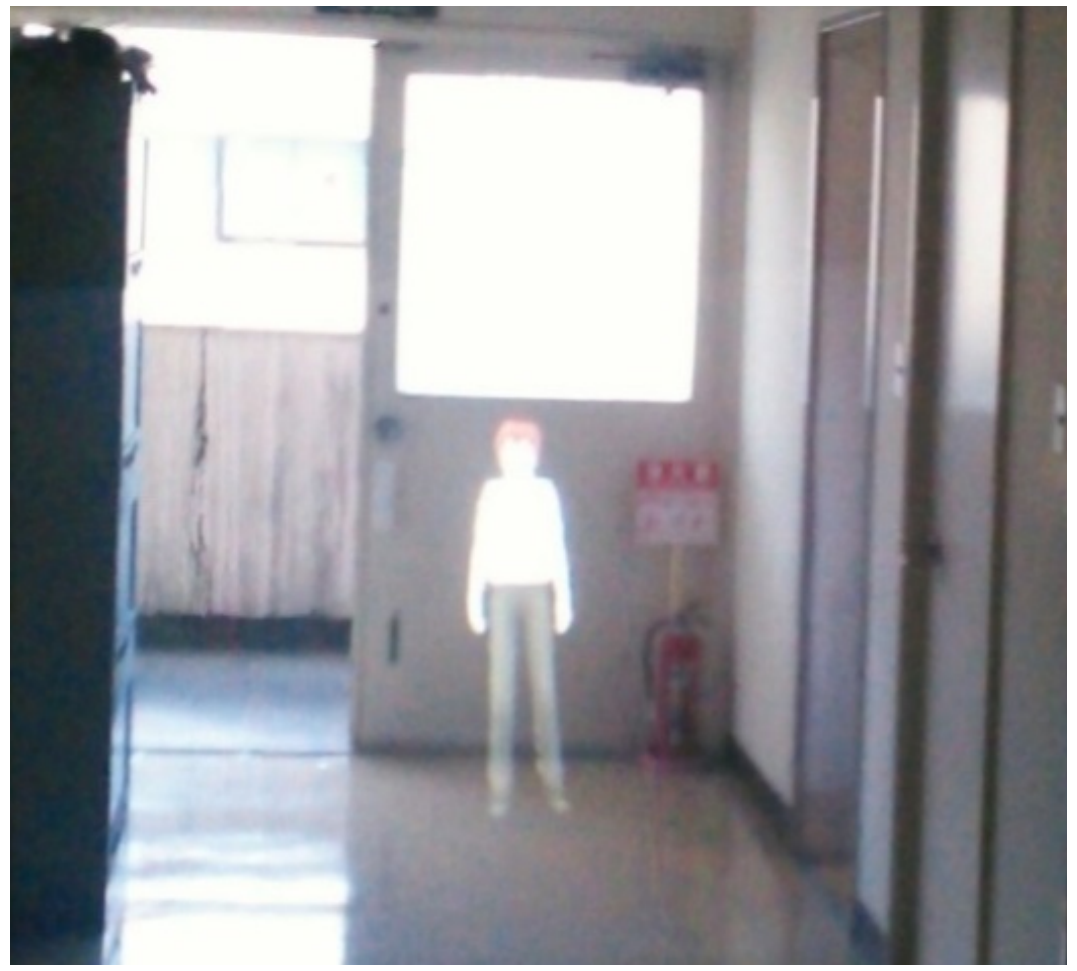

AR View (Superimposed virtual student)

Fig. 6. Screenshots of System-3 
Paper-Using Digital Game, Augmented Reality, and Head Mounted Displays for Immediate-Action Commander Training

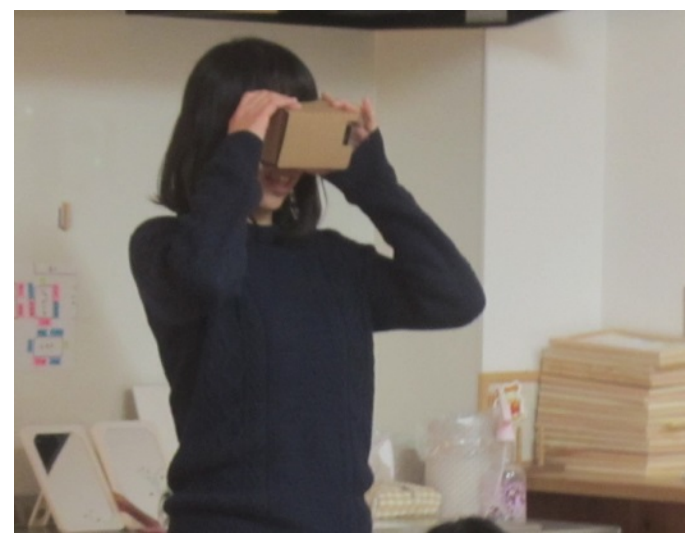

System-2 in use

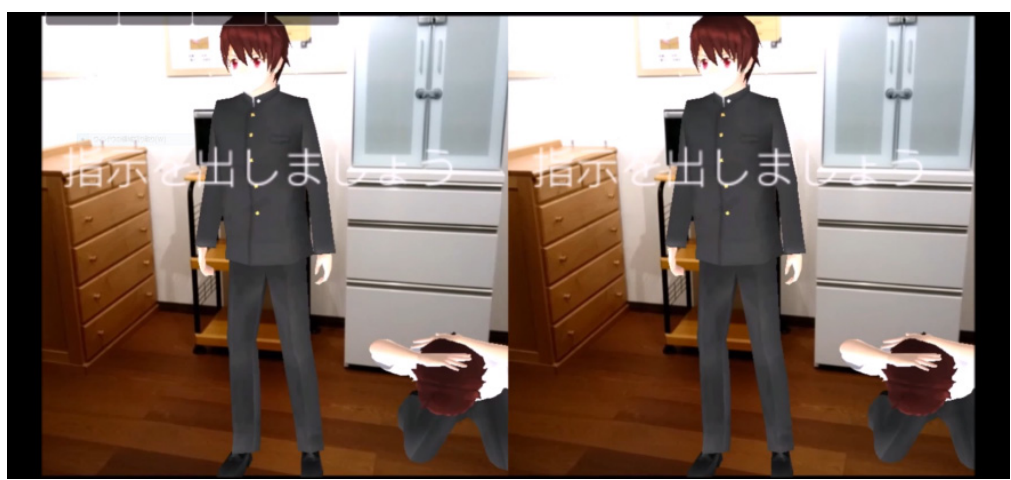

Stereoscopic Screenshots (two children in earthquake) Superimposed message: "Say a proper command."

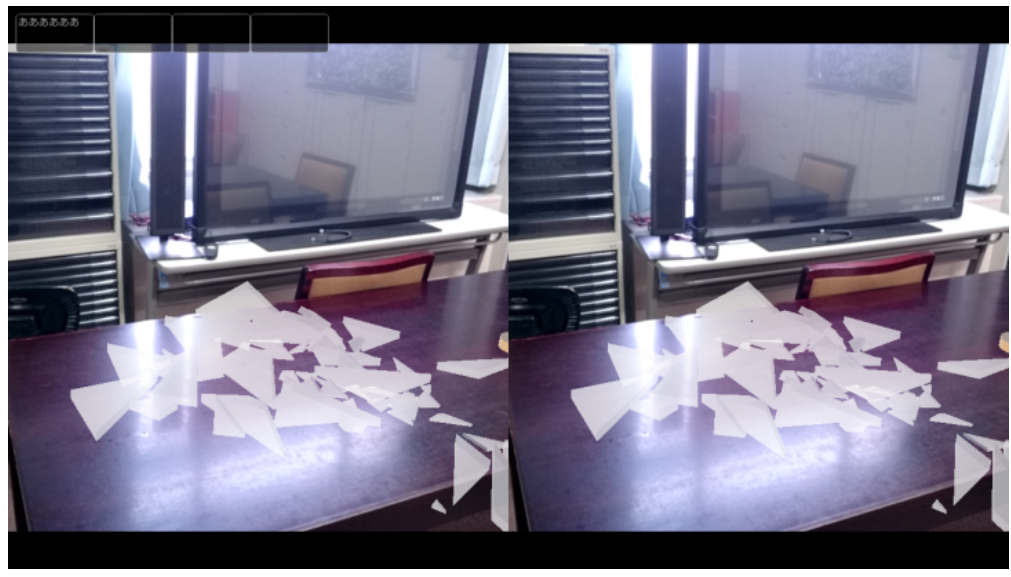

Fragments of broken glass

Fig. 7. Snapshot and screenshots in experimental use 


\section{Experimental Use}

Experimentally, we used System-2 in an IACer training program for preschool teachers. Fig. 7 shows the experimental snapshot and screenshots of System-2. The used IF scenario, which dealt with earthquake and had no branching, comprised the following sequential situations.

(1) Emergency earthquake warning: When two children chat in a playroom without tables, an emergency earthquake warning suddenly sounds. At this time, a teacher (trainee) must vocally command the children to take an appropriate immediate action (for example, "Move away from the window!").

(2) Long massive shake: Several seconds later, an intense shake begins, i.e., System-2 shakes the real-time vision to represent the earthquake and superimposes fragments of broken glass with a loud crash (sound effect). The teacher must command, "Huddle on the floor and protect your head!" One child takes the appropriate immediate action, whereas the other remains standing. In such a situation, the teacher must repeat the same command for this child and additionally offer encouraging words to calm down their nerves.

(3) Evacuation: When the shake stops, one child keeps huddling on the floor and does not start evacuation whereas the other tries to bolt for the door. In this situation, the teacher must command, "Let's evacuate outside calmly!" Consequently, the two children come out of the playroom safely.

\section{Comparative Survey}

We conducted a comparative brief questionnaire to evaluate the visual reality and usability of the three systems without the voice-based interactions. The participants (23 university students) used the systems in a fixed IF scenario. For each system, they answered the following questions: "Did you feel visual reality?" (1 to 5 on a 5 -scale Likert) and "Which systems (HMDs) were easiest to use?" (multiple-choice questions). The mean values for the former question were 4.04, 3.83, and 3.01 for System1, System-2, and System-3, respectively. With regard to the visual reality, System-1 (Oculus Rift) and System-2 (Google Cardboard) are superior to System-3. This result may be caused by HMDs' viewing angles. The responses for the latter question were 0,22 , and 5 for System-1, System-2, and System-3, respectively. With regard to the usability, System-2 is decisively superior to System-1 and System-3. This result may be caused by HMD's wearability (e.g., weight and fit). Overall, from these results, we believe System-2 to be the most appropriate option for the IACer training program.

\section{Conclusions}

IACers can protect children's lives. As technology-enhanced IACer training programs, we prototyped three AR systems: System-1, System-2, and System-3 corresponding to a binocular opaque HMD, a smartphone-based binocular opaque HMD, 
and an optical see-through HMD, respectively. These systems aim at improving situational and audio-visual realities by superimposing virtual objects (e.g., 3DCG characters) onto a real-time vision or a trainee's actual view according to an interactive fiction (IF) scenario. A branched IF scenario that provides the interactivity (the game element) will be able to control voice-based interactions between the virtual objects and the trainee.

We have not realized voice-based interactions and did not improve the visual reality in terms of photometric consistency, which means that the AR systems are still inprogress. Through the comparative survey, we believe that System-2 has the most promising practical system for technology-enhanced IACer training programs. Therefore, we will continue to mainly focus on System-2 and deal with the unrealized tasks. Additionally, we intend to implement a training-reflection system (e.g., replaying the recorded AR views with subtitles of the recognized vocal commands) and additional game elements (e.g., giving scores to trainees who succeed in giving vocal commands or quickly reach to a good end).

In the future, we must evaluate the systems in more detail. In disaster education, it is particularly important to consider what to evaluate and how to evaluate it. The realities as well as the educational effectiveness of the systems should be evaluated through large-scale training practices. We will make a maximal effort to establish IACer training programs as quickly as possible prior to the occurrence of serious disasters.

\section{References}

[1] M.H. Tsai, M.C. Wen, Y.L. Chang, and S.C. Kang, "Game-based education for disaster prevention," AI \& Society, vol. 30, no. 4, pp. 463-475, 2014. https://doi.org/10.1007/s00146-014-0562-7

[2] I. Dunwell, P. Petridis, S. Arnab, A. Protopsaltis, M. Hendrix, and S. Freitas, "Blended game-based learning environments: Extending a serious game into a learning content management system," Proc. of Third International Conference on Intelligent Networking and Collaborative Systems (INCoS), pp. 830-835, 2011. https://doi.org/10.1109/incos.2011.58

[3] N. Capuano, and R. King, "Knowledge-based assessment in serious games: An experience on emergency training," Journal of e-Learning and Knowledge Society, vol. 11, no. 3, pp. 117-132, 2015.

[4] D.D. Djordjevich, P.G. Xavier, M.L. Bernard, J.H. Whetzel, M.R. Glickman, and S.J. Verzi, "Preparing for the aftermath: Using emotional agents in game-based training for disaster response," Proc. of 2008 IEEE Symposium on Computational Intelligence and Games, pp. 266-275, 2008. https://doi.org/10.1109/CIG.2008.5035649

[5] D. Wahyudin, and S. Hasegawa, "Mobile serious game design for training ethical decision making skills of inexperienced disaster volunteers," The Journal of Information and Systems in Education, vol. 14, no.1, pp. 28-41, 2015. https://doi.org/10.12937/ejsise.14.28

[6] M.E.C. Santos, A. Chen, T. Taketomi, G. Yamamoto, J. Miyazaki, and H. Kato, "Augmented reality learning experiences: Survey of prototype design and evaluation," IEEE Transactions on Learning Technologies, vol. 7, no. 1, pp. 68-56, 2014. https://doi.org/10.1109/TLT.2013.37 
[7] J. Bacca, S. Baldiris, R. Fabregat, S. Graf, and Kinshuk, "Augmented reality trends in education: A systematic review of research and applications," Educational Technology \& Society, vol. 17, no. 4, pp. 133-149, 2014.

[8] S. Nilsson, B.J.E. Johansson, and A. Jönsson, "Cross-organizational collaboration supported by augmented reality," IEEE Transactions on Visualization and Computer Graphics, vol. 17, no. 10, pp.1380-1392, 2010.

[9] D. Datcu, S. Lukosch, J. Lukosch, and M. Cidota, "Using augmented reality for supporting information exchange in teams from the security domain," Security Informatics, vol.4, no. 10, 2015. https://doi.org/10.1186/s13388-015-0025-9

[10] T. Reiners, H. Teräs, V. Chang, L. Wood, S. Gregory, D. Gibson, N. Petter, and M. Teräs, "Authentic, immersive, and emotional experience in virtual learning environments: The fear of dying as an important learning experience in a simulation," Teaching and Learning Forum 2014, 2014.

[11] A. Suarez, S. Ternier, M. Kalz, and M. Specht, "Supporting inquiry-based learning with Google Glass (GPIM)," Interaction Design and Architecture(s) Journal (IxD\&A), vol. 24, pp. 100-110, 2015.

[12] B. Wang, H. Li, Y. Rezgui, A. Bradley, and H.N. Ong, "BIM based virtual environment for fire emergency evacuation,” The Scientific World Journal, vol. 2014, 2014

[13] J. Kawai, H. Mitsuhara, and M. Shishibori, "Tsunami evacuation drill system using smart glasses,” Procedia Computer Science, vol.72, pp.329-336, 2015. https://doi.org/10.1016/ j.procs.2015.12.147

[14] H. Mitsuhara, J. Kawai, K. Iguchi, and M. Shishibori, "Game-based evacuation drills using simple augmented reality," Proc. of the 16th IEEE International Conference on Advanced Learning Technologies - ICALT2016, pp.133-137, 2016. https://doi.org/10.1109/icalt. 2016.71

[15] J. Kawai, H. Mitsuhara, and M. Shishibori, "Game-based evacuation drill using augmented reality and head-mounted display," Interactive Technology and Smart Education, vol. 13, issue 3, pp.186-201, 2016. https://doi.org/10.1108/ITSE-01-2016-0001

[16] J. Fischer, W. Jiang, and S. Moran, "AtomicOrchid: A mixed reality game to investigate coordination in disaster response," Proc. of the 11th International Conference on Entertainment Computing (ICEC 2012), pp. 572-577, 2012. https://doi.org/10.1007/978-3-64233542-6 75

[17] K. Meesters, and B.A Van de Walle, "Disaster in my backyard: A serious game introduction to disaster information management," Proc. of the 10th International Conference on Information Systems for Crisis Response and Management (ISCRAM 2013), pp. 145-150, 2013.

\section{Authors}

H. Mitsuhara received the B.E. and M.E. degrees from Kindai University in 1998 and 2000, and then he received the Ph.D. degree from Tokushima University in 2003. $\mathrm{He}$ is currently an associate professor at Graduate School of Science and Technology, Tokushima University, Tokushima, Japan. His research interests include game-based education, entertainment computing, human-computer interaction, web-based learning, and disaster education. (e-mail: mituhara@is.tokushima-u.ac.jp).

K. Iguchi received the B.E. degree from Tokushima University, Japan. He is currently a master's degree student at Graduate School of Advanced Technology and 
Paper-Using Digital Game, Augmented Reality, and Head Mounted Displays for Immediate-Action Commander Training

Science, Tokushima University, Tokushima, Japan. His research interests include AR and HMDs for disaster education.

M. Shishibori was born in 1965. He received his BS Degree in 1991, his MS Degree in 1993 and PhD Degree in 1997, from Tokushima University, Japan. He is currently a Professor in the Department of Computer Science, Graduate School of Science and Technology, Tokushima University, Japan. His research interests include multimedia processing, information retrieval, and natural language processing.

This study was supported by a Grant-in-Aid for Scientific Research (C) No. 15K01026 from the Japan Society for the Promotion of Science. Article submitted 26 September 2016. Published as resubmitted by the authors on 13 December 2016. 